\title{
Microscopic Characterizations of Upconversion-Induced Near-Infrared Light Harvest in Hybrid Perovskite Solar Cells
}

\author{
Mathilde Schoenauer Sebag ${ }^{1}$, Zhelu $\mathrm{Hu}^{1}$, Karmel de Oliveira Lima², Patrick Gredin ${ }^{2,3}$, Michel Mortier², \\ Laurent Billot ${ }^{1}$, Lionel Aigouy ${ }^{1}$, and Zhuoying Chen ${ }^{1, *}$ \\ ${ }^{1 .}$ LPEM, ESPCI Paris, PSL Research University, Sorbonne Université, CNRS, Paris, France. \\ 2. Institut de Recherche de Chimie Paris, Chimie Paris Tech, PSL Research University, CNRS, Paris, \\ France. \\ 3. Sorbonne Université, Faculté des Sciences, UFR 926, Paris, France. \\ * Corresponding author: zhuoying.chen@espci.fr
}

Photon upconversion represents a promising avenue to reduce the spectral mismatch losses limiting the efficiency of solar cells [1-3]. Spectral mismatch losses, also called sub-bandgap losses, originate from the fact that photons of energies below the absorption threshold of the photovoltaic absorber cannot be absorbed and cannot contribute to the current generation in the solar cell. Such losses can be quite significant: For single-junction crystalline silicon solar cells with a bandgap of $1.1 \mathrm{eV}$, sub-bandgap losses account for more than 19\% of the power incident from the sun [2]. This percentage is even more elevated, reaching 39\%, for solution-processed organic-inorganic hybrid perovskite halide solar cells due to their larger bandgap (e.g. about $1.5 \mathrm{eV}$ for methylammonium lead iodide perovskite) [2]. Various forms of upconversion materials have therefore been applied into solar cells based on different semiconducting materials [4-7]. While these results are highly encouraging, the application of upconversion NPs (UCNPs) in the front side of the solar cell (before the illumination reaches the semiconducting layer) can render the mechanistic study very complex due to the possible structural/morphological modification of the absorber materials deposited on top and the optical scattering induced by the UCNPs. Most current studies are based on current-voltage characterizations, a more detailed photovoltaic-microscopic correlation characterization would be valuable to reveal the intrinsic upconversion contribution.

In this presentation, we investigate in detail macroscopically and microscopically the impact of inserting upconversion nanoparticles (UCNPs) into a functional solar cell based on solution-processed mixed-cation lead mixed-halide perovskite $\mathrm{FA}_{0.83} \mathrm{Cs}_{0.17} \mathrm{~Pb}\left(\mathrm{I}_{0.6} \mathrm{Br}_{0.4}\right)_{3}$. Uniform and well-crystalline $\mathrm{Yb}^{3+} / \mathrm{Er}^{3+}$ co-doped $\mathrm{KY}_{7} \mathrm{~F}_{22} \mathrm{UCNPs}$ are applied onto either the FTO/perovskite or the perovskite/HTL interface of a perovskite solar cell (the front- or rear-side configuration) while the density of UCNPs are controlled by their concentration in solution during the deposition. By comparison to the control device, power conversion efficiency (PCE) enhancement will be studied both in the front- and rear-side NP insertion configuration with an optimal UCNP solution concentration. To quantify the upconversion contribution, microscopic solar cell characterizations by the light-beam induced current (LBIC)/fluorescence mapping technique are carried out on devices with only half of their perovskite/hole transport layer (HTL) interface decorated by UCNPs fabricated on purpose. These mapping experiments offered a detailed microscopic picture allowing a correlation of the electrical and optical contribution of UCNPs together with the solar cell morphology. The optical contribution of the UCNPs in a perovskite solar cell will be discussed in detailed.

\section{References:}

[1] B.M. van der Ende, L. Aarts and A. Meijerink, Phys. Chem. Chem. Phys. 11 (2009), p. 11081.

[2] J.C. Goldschmidt and S. Fischer, Adv. Opt. Mater. 3 (2015), p. 510. 
[3] J. de Wild et al., Energy Environ. Sci. 4 (2011), p. 4835.

[4] M. He et al., Angew. Chemie Int. Ed. 55 (2016), p. 4280.

[5] H.Q. Wang et al., Adv. Mater. 23 (2011), p. 2675.

[6] S. Fischer et al., J. Appl. Phys. 108 (2010), p. 044912.

[7] S. Chen et al., Mater. Lett. 77 (2012), p. 17.

(a)

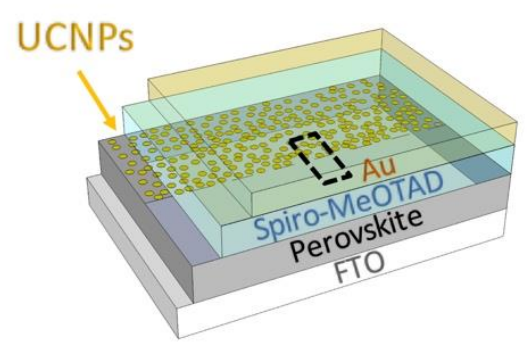

(e)

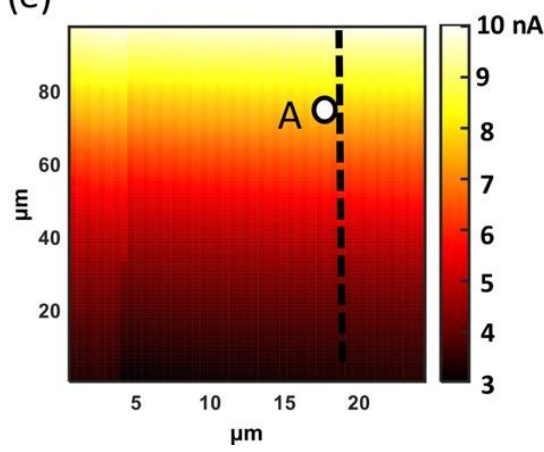

(b)

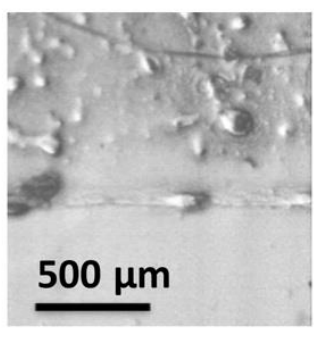

(c)

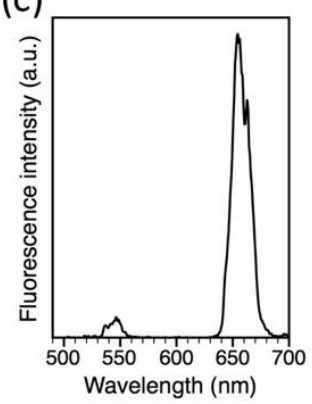

(d)

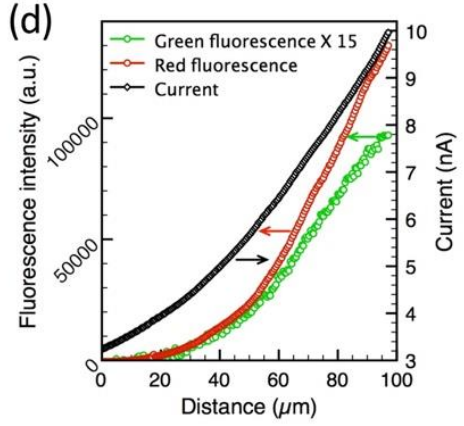

(f)

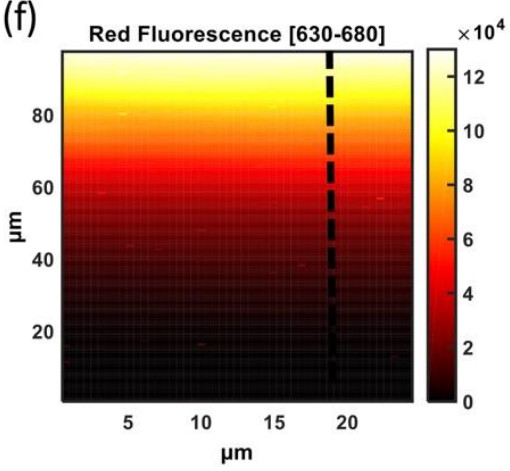

(g)

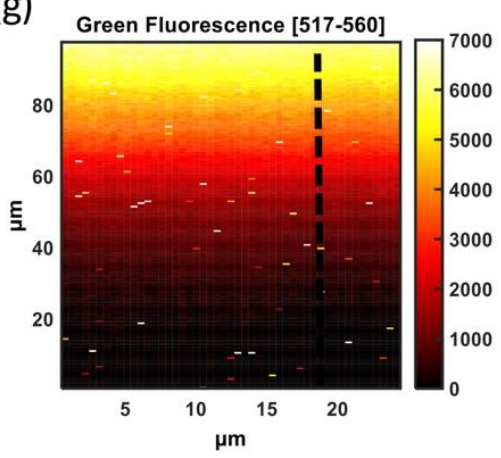

Figure 1. (a) Schematic (not to scale) of the UCNP-incorporated perovskite solar cell structure where half of the perovskite/HTL interface was decorated by UCNPs. The NIR laser $(975 \mathrm{~nm})$ was focused at the perovskite/HTL interface through the FTO side. The dark dashed square represents the device area where LBIC/fluorescence mapping was carried out. (b) Optical image of the top-view (imaged from the gold electrode side) of the UCNP-incorporated perovskite solar cell. The interface between the part with and without UCNPs is clearly visible. (c) The upconversion fluorescence spectrum recorded during the LBIC mapping at the point A (labelled in (e)). (d) $J_{s c}$ and fluorescence line profile of the dashed line shown in (e), (g) and (h). (e) Short-circuit current $J_{s c}$ mapping under the excitation of a $975 \mathrm{~nm}$ NIR laser. (f) and (g) The red and green fluorescence mapping recorded simultaneously during LBIC mapping. Each pixel represents the fluorescence signal integrated between $630 \mathrm{~nm}$ and $680 \mathrm{~nm}$ for red fluorescence (f) and between $517 \mathrm{~nm}$ and $560 \mathrm{~nm}$ for green fluorescence $(\mathrm{g})$. 